Jens Schröter

\title{
Cultures of Pattern Recognition in Particle Physics. \\ Preliminary Remarks.
}

[PRE PRINT]

In recent years, there has been increased attention to the role of artificial intelligence (AI) methods in the natural and social sciences. AI today means mainly different methods of machine learning. Pattern recognition, that is finding structures in large amounts of highly complex data, is one of the central usages of machine learning. ${ }^{1}$ So we can assume that its role in science is also pattern recognition, that is to find structures in complex data. And at least for natural sciences, where data play a different role than in, let's say, the humanities, this is true. In the following paper I want to discuss the role of machine learning in particle physics, focused on one example, the spectacular discovery of the Higgs-boson in 2012. ${ }^{2}$ To understand more clearly the role of machine learning and if and how it changed practices and cultures of pattern recognition science, it is necessary to take a look into the history of particle physics, to 1964 - the discovery of the so-called ' $\Omega^{-}$'.

\footnotetext{
${ }^{1}$ See Ethem Alpaydin, Machine Learning. The New AI, (Cambridge, MA: MIT Press, 2016), chapter 3.

${ }^{2}$ In Peter Galison, Image and Logic. A Material Culture of Microphysics (Chicago: University of Chicago Press 1997 ) the role of machine learning is not mentioned (artificial intelligence only four times) - but the study is from 1997. Research remains to be done.
} 


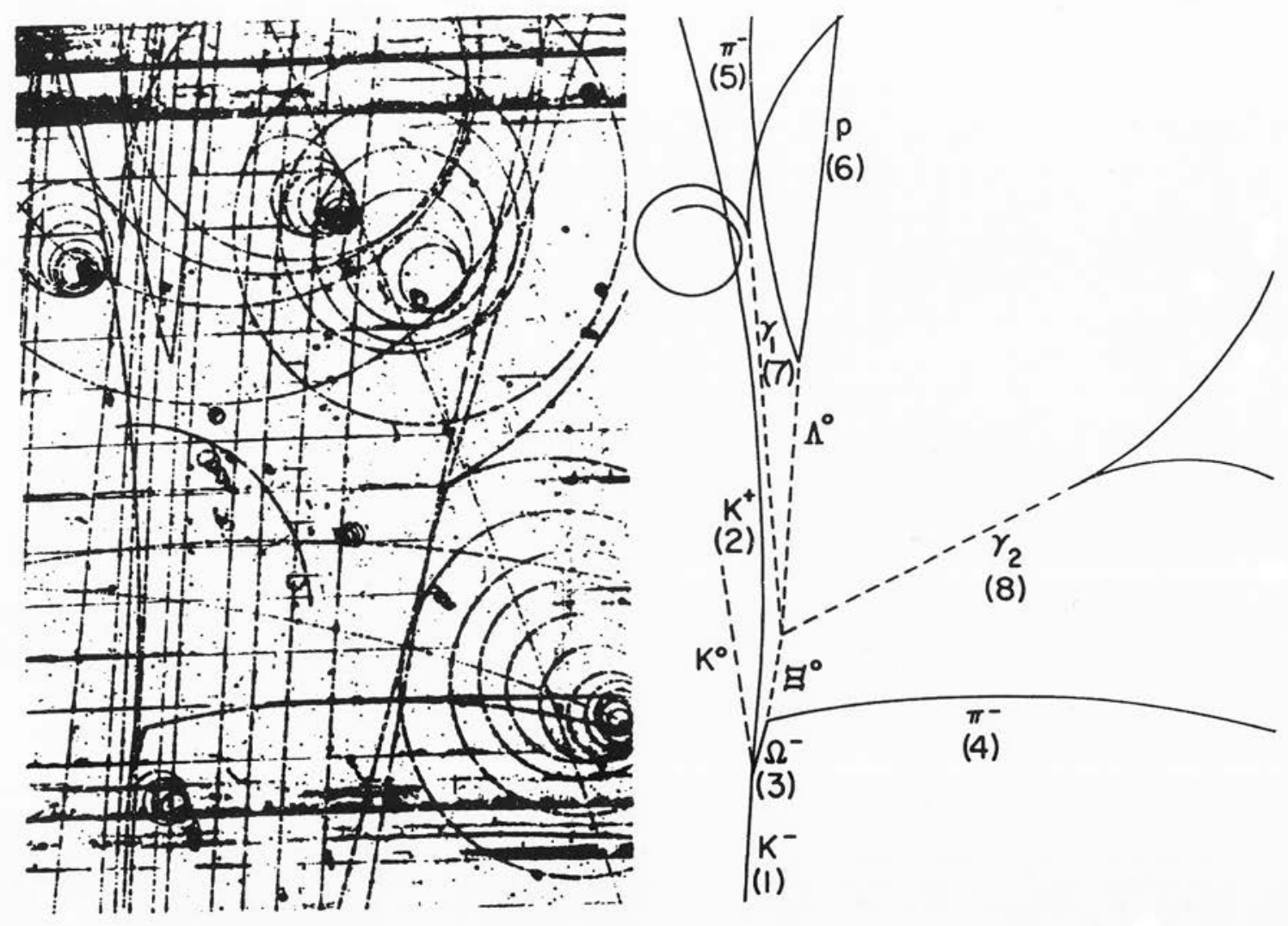

Fig. 1

Photography of Omega Minus-Event with explaining 'line diagram', from: V. E. Barnes et al., 'Observation of a Hyperon with Strangeness Minus Three', in: Physical Review Letters, Vol. 12, No. 8 (24.2.1964), pp. 204-206 (205, processed).

This image shows one of the most important photos from the history of particle physics, a golden event, as Peter Galison has called it. ${ }^{3}$ If one looks at the photo in a decontextualized way, it is unrecognizable. One cannot say what is depicted - because reference depends on knowledge. ${ }^{4}$ In the context of the commenting line diagram, such as it is printed in the (nowadays legendary) publication of $1964^{5}$, the image becomes meaningful, the pattern can be recognized. The sheer complexity of traces can be reduced. The photograph is combined with a line diagram that shows which traces are important and which are noise. The little kink at the bottom is the short trace of the $\Omega^{-}$. We can see how it decays. Symbols are added to the lines, which make - at least for experts - clear which traces of what particles are

\footnotetext{
${ }^{3}$ Ibid., 22/23.

${ }^{4}$ See Catherine Elgin, With Reference to Reference (Indianapolis: Hackett 1983).

${ }^{5}$ See V. E. Barnes et al., "Observation of a Hyperon with Strangeness Minus Three," Physical Review Letters 12 , no. 8 (24.2.1964): 204-206.
} 
represented. Interestingly, neutral particles are invisible in the photograph, their traces have to be inferred from the overall pattern, which is constructed by the diagram. The numbers in parentheses point to additional information in the text. More detailed arguments for the interpretation of the traces are given there.

The photograph is photograph number 97,025 of a series of experiments conducted at the Brookhaven Laboratory, New York. ${ }^{6}$ In the American laboratory for particle physics, researchers had set up a bubble chamber filled with approximately 1,000 litres of hydrogen. A beam of negatively charged kaons was shot into the laboratory's 80 -inch tall bubble chamber. During the collisions of the beam with the atoms, numerous new particles arose which, dependent on their charge and their mass, left behind traces resembling tiny condensation trails. But only one particle interested the researchers - namely the particle with the name ' $\Omega$ '. Murray Gell-Mann was the first person to assert the existence of this particle as early as 1962 to his colleagues at an international conference. Gell-Mann could predict the particle and its precise characteristics, since he had discovered a new regularity in the previously rather confusing 'particle zoo', namely the so-called 'SU(3) symmetry'?

This theory was 'confirmed' by the 'evidence' of the traces on photo number 97,205 from 1964. The simple fact that it was the 97,205th image on which the sought-after traces occurred demonstrates how much effort had been put into the particle chase. A film was passed at high speed over the bubble chamber in order to capture the countless and disorganized collision events. After the bombardment had ended, the film had to be evaluated one image at a time. For this purpose there were entire teams of specialists (called 'scanners') who looked out for suspicious traces. If pieces of photographic evidence were found, they then had to be interpreted with the use of digital computers. This was a highly complex process, which was (in a general form) described in detail in a 1967 paper with the title "Man-Machine Cooperation in Digital Pattern Recognition of Bubble Chamber Tracks". The interesting point here is of course, that the - nowadays so popular - notion of "pattern

\footnotetext{
${ }^{6}$ See Nicholas Samios, "Early Baryon and Meson Spectroscopy Culminating in the Discovery of the Omega Minus. SU(3) and Quarks", available at www.osti.gov/bridge/servlets/purl/10162083-fAD7aQ/10162083.PDF. See also Nicholas Samios, "Early Baryon and Meson Spectroscopy Culminating in the Discovery of the Omega-Minus and Charmed Baryons," in The Rise of the Standard Model. Particle Physics in the 1960s and 1970s, ed. Lilian Hoddeson et al. (Cambridge, NY: Cambridge University Press 1997), 525-541.

${ }^{7}$ On the history of quark physics see Andrew Pickering, Constructing Quarks. A Sociological History of Particle Physics (Chicago: University of Chicago Press 1984). See also Laurie M. Brown et al., "The Rise of the Standard Model: 1964-1979," in Hoddeson et al., 3-35. See also Wolfgang Hagen, "Das atlantische Standardmodell - Anmerkungen zu einer Genealogie der Elektrizität und ihrer Medien", available at http://www.whagen.de/PDFS/07953_HagenDasatlantischeStanda_2014.pdf.

${ }^{8}$ Cf. R. C. Strand, "Man-Machine Cooperation in Digital Pattern Recognition of Bubble Chamber Tracks," Annals of the New York Academy of Sciences 157, no. 1 (1967): 65-82.
} 
recognition' was already used at that time to describe the discovery of predicted structures in the bulk of photographic data. ${ }^{9}$ This pattern recognition proceeded along a complex "operational chain" $" 10$ of translations between different media and their correlated practices.

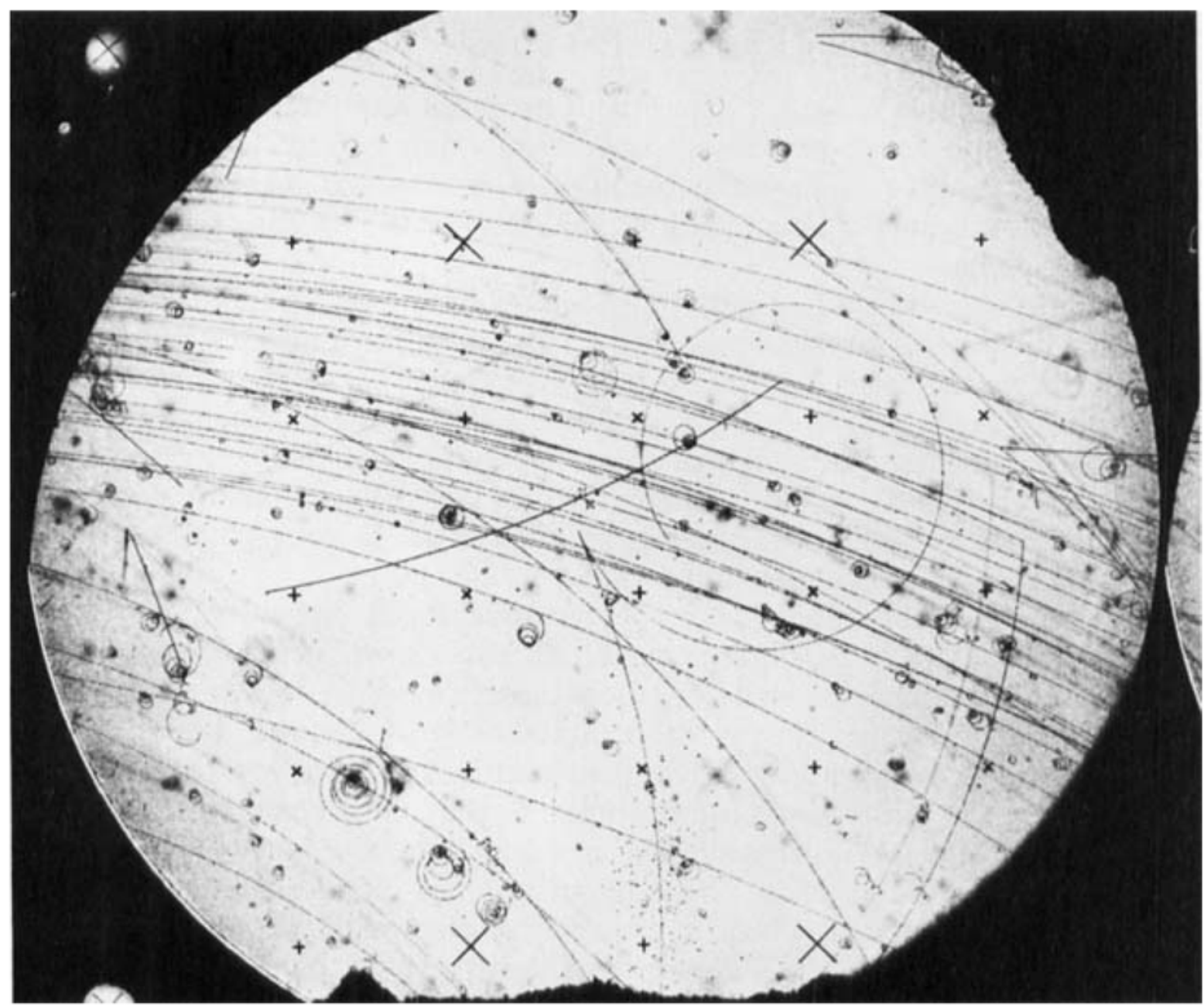

FIGURE 1. Brookhaven National Laboratory 30-inch bubble chamber photograph.

Fig. 2

Bubble chamber photography, Brookhaven Lab, R. C. Strand, "Man-Machine Cooperation in Digital Pattern Recognition of Bubble Chamber Tracks," Annals of the New York Academy of Sciences 157, no. 1 (1967): 65-82 (66).

Fig. 2 shows a raw bubble chamber photograph. The production of such images was quite complicated. Since to the speed of the appearances in the bubble chamber the films had to move at very high speeds too - what lead to problems, e.g. films getting stuck etc. The

\footnotetext{
${ }^{9}$ See on the notion of "photographic data" Estelle Blaschke, "From Microform to the Drawing Bot: The Photographic Image as Data," Grey Room 75 (2019): 60-83.

${ }^{10}$ See Bruno Latour, "The 'pedofil' of Boa Vista: A Photo-Philosophical Montage," Common Knowledge 4, no. 1 (1995): 144-187.
} 
complexity of the traces in each single frame is overwhelming, especially since it was known that neutral particles don't leave traces: "they present a challenging pattern recognition problem for the skilled observer". ${ }^{11}$ It has to be interpreted - and the crosses seen in the picture help to adjust its recording and reading. They were used to calibrate the images. Because the processes were quite fast, lots of pictures were taken: "When the bubbles have grown to optimal size, a timed light flash images the tracks on film. A serial picture number is also recorded. Three stereo-cameras are used. Each camera is fed from a reel of about $1,000 \mathrm{ft}$. of film with room for a few thousand exposures depending on the length of the chamber image. At Brookhaven what we call one roll of bubble chamber film is composed of three reels of film. As many as 50 to 500 thousand stereo-pictures may be required for an experiment; and as many as 250 thousand pictures can be taken per month." 12

TABLE 1

Operations Involving Experimental Data and Their Associated Hardware for a BubBLE CHAMBER EXPERIMENT

\begin{tabular}{|c|c|}
\hline Experimental Data & Hardware \\
\hline Prepare initial state ........ & $\begin{array}{l}\text { Accelerator beam } \\
\text { Bubble chamber }\end{array}$ \\
\hline Store all final states. & Stereophotography film \\
\hline Event extraction & Scanning machine \\
\hline Extraction of precision track points ............ & Measuring machine \\
\hline Processing & Digital computer \\
\hline Display for physics analysis & Digital computer \\
\hline
\end{tabular}

Fig. 3

Table of necessary steps for the analysis of the images - for the production of pattern recognition, Brookhaven Lab, R. C. Strand, "Man-Machine Cooperation in Digital Pattern Recognition of Bubble Chamber Tracks," Annals of the New York Academy of Sciences 157, no. 1 (1967): 65-82 (69).

Fig. 3 informs about the usual flow of steps in producing the desired pattern recognition and finding the relevant images. The left side of the table shows what operations are performed on the data, the right side shows the correlated 'hardware' (media technology), to store, process and transmit information. Interestingly, the left row of the table by specifying

\footnotetext{
${ }^{11}$ Strand, Man-Machine Cooperation, 76.

${ }^{12}$ Strand, Man-Machine Cooperation, 66.
} 
the operations shows different types of practices. It would be very helpful to learn more about the training procedures that were used to train the scientists in the lab to perform these practices like extraction, processing etc. ${ }^{13}$

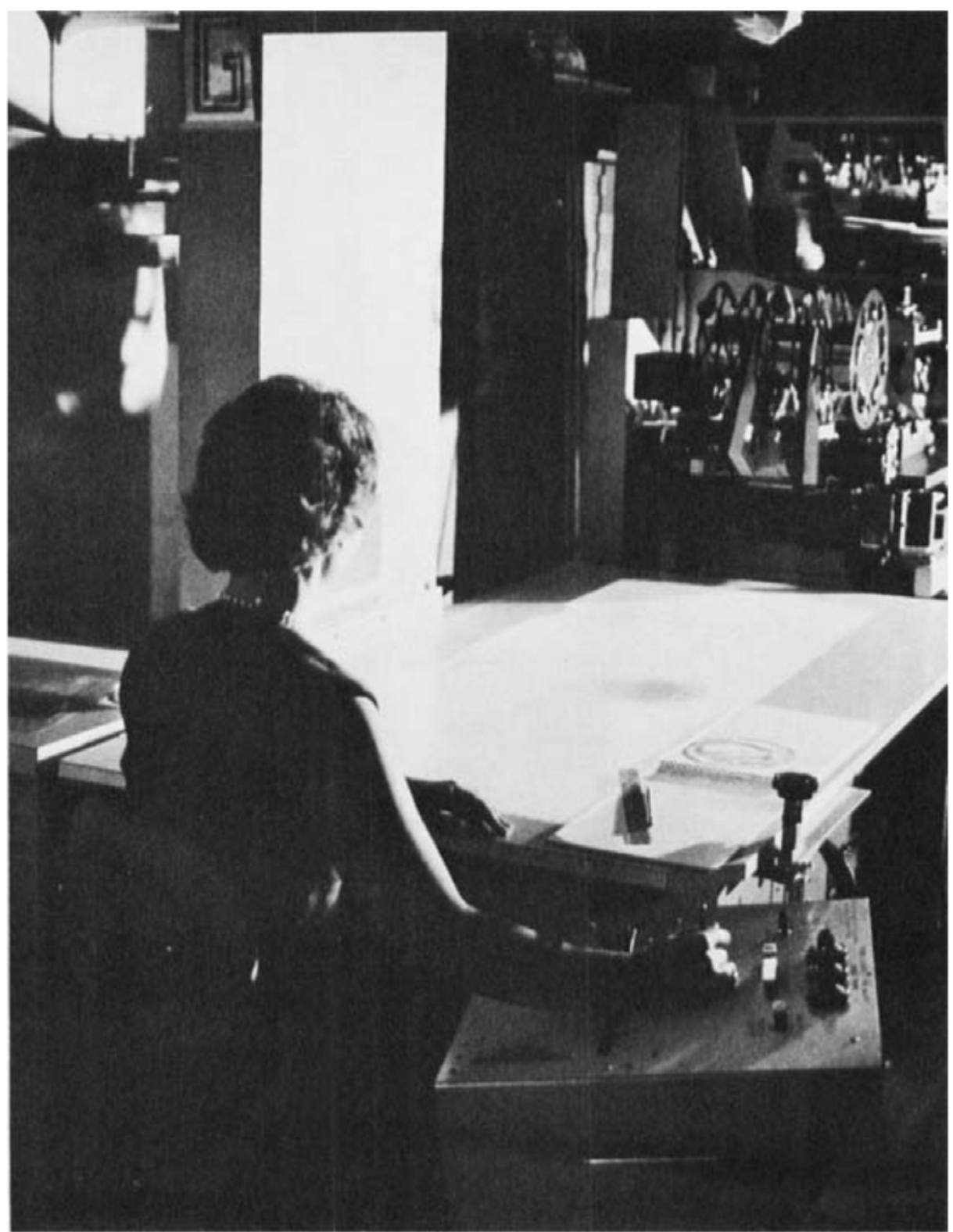

Figure 4. A bubble chamber film scanning table.

\footnotetext{
${ }^{13}$ See Peter Galison, "Judgment Against Objectivity.” In Picturing Science, Producing Art, ed. Caroline A. Jones and Peter Galison (New York: Routledge 1998), 327-359 on the central role of the trained eye and the "interpreted image" (329).
} 


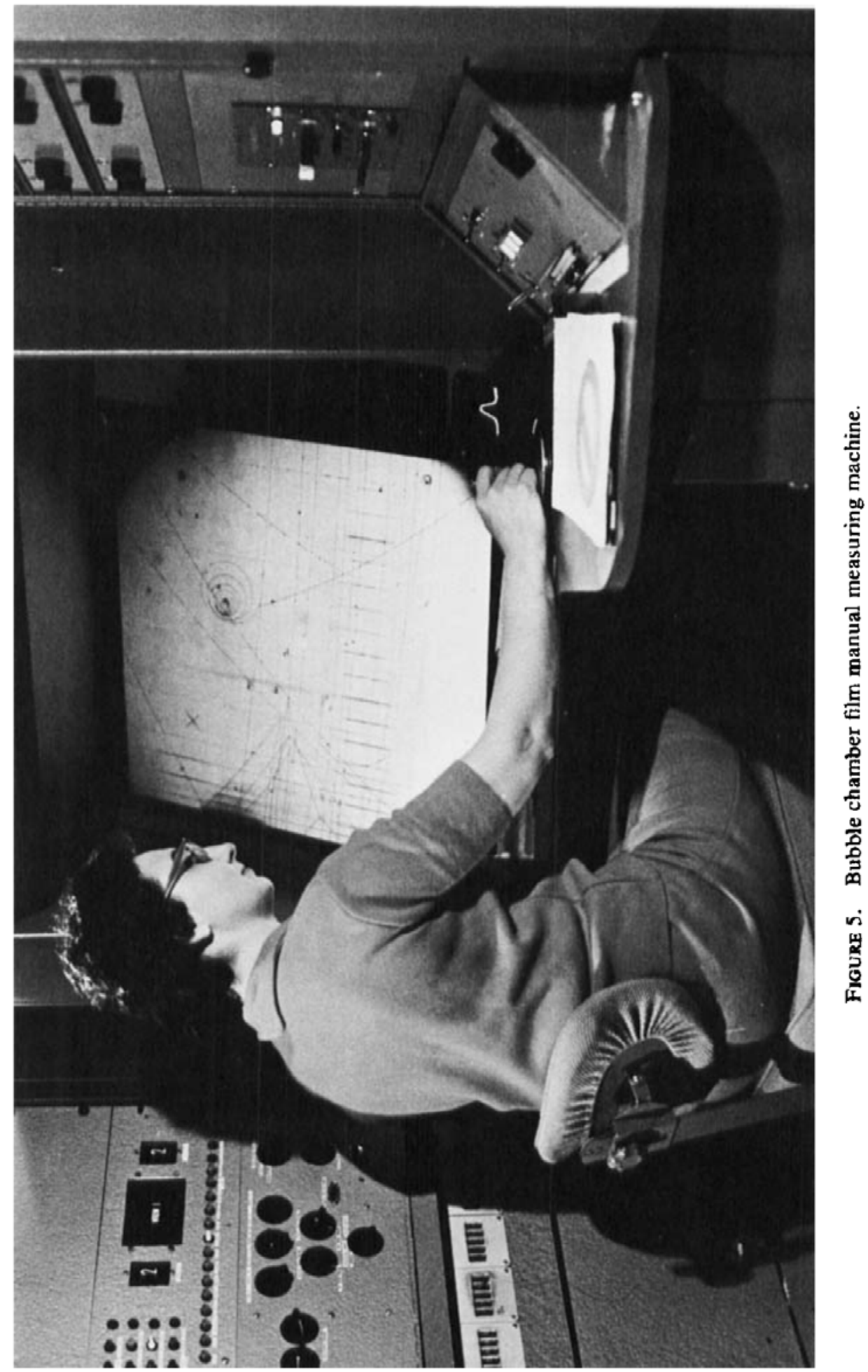

Fig. 4 a, b

Bubble chamber film measurement, R. C. Strand, "Man-Machine Cooperation in Digital Pattern Recognition of Bubble Chamber Tracks," Annals of the New York Academy of Sciences 157, no. 1 (1967): 65$82(70,75)$. 
Fig. 4 a, b show the initial steps of the selection and analysis. All the images had to be checked, one by one, by specially trained operators - mostly women, the culture of pattern recognition is obviously gendered. The pictures were all taken, as mentioned above, in stereo to give a clearer picture of the spatial orientation of the tracks. This shows that technologies like stereophotography, which were popular mass media in the late $19^{\text {th }}$ century, do not disappear around 1900, as Jonathan Crary put it famously when he spoke about the "collapse of the stereoscope."14 It disappeared as a popular medium from the living rooms, because from 1889 onward one could take photographs oneself, send photo-postcards, or somewhat later go to the movies (where stereoscopy sometimes re-emerged). But stereoscopy does not disappear in general. Crary is focusing only on the use of three-dimensional image technology as popular mass media without acknowledging their increasingly important role within the diverse scientific (or military) practices. ${ }^{15}$ In certain cultures of pattern recognition seemingly outmoded technologies can be reused for new purposes. Instead of spectacularly presenting spatial depth for the purposes of entertainment, stereoscopy became important to reveal spatial patterns for the production of knowledge. But the stereoscopic images in the culture of pattern recognition at the Brookhaven lab had to be understood and 'read' correctly. The women were trained to 'see' interesting traces. This training is unfortunately not very good explored.

Moreover, it is highly important that the pattern recognition in particle physics was one of the first places where images were digitalized to analyze them better ${ }^{16}$ : "A mechanically deflected flying spot digitizer, FSD, was built to extract a bubble image centered digital representation of one stereo view. A uniform raster scan is achieved by moving the film on a precision stage past the fixed line of the flying spot. [...] Each bubble image center is located with a precision of a few microns. To the FSD, a typical view might contain an average of 25 points per 2,000 scan lines spaced 50 microns apart for a total of 50,000 points. During the scan, the data are transmitted directly to an IBM 7094 computer for processing. An orthogonal scan mode is provided for those tracks which make an angle greater

\footnotetext{
${ }^{14}$ Jonathan Crary, Techniques of the Observer. On Vision and Modernity in the Nineteenth Century (Cambridge, MA: MIT Press 1990), 127.

${ }^{15}$ See Jens Schröter, 3D. History, Theory and Aesthetics of the Technical-transplane Image (New York et al.: Bloomsbury 2014).

${ }^{16}$ See Jens Schröter, "Das Ende der Welt. Analoge vs. Digitale Bilder - mehr und weniger Realität?," in Analog/Digital. Opposition oder Kontinuum?, ed. Jens Schröter and Alexander Böhnke (Bielefeld: transcript 2004), 335-354 on the early history of digital imaging - which unfortunately forgot the early work done in particle physics. See Götz Hoeppe, "Medium, Calculation, Play: On Digital Images in Scientific Practice," Social Studies of Science, Vol. 49, No. 5 (2019): 758-784 for a recent take on digital imaging in scientific practices, attempting "to link the microstructure of media to their communicative uses." (758)
} 
than $45^{\prime}$ to the beam direction [...] The first semi-automatic FSD data extraction operation is a manual operation. Film for the FSD is prescanned. A further reduction in the magnitude of the digital data to be processed is achieved by the scanning technician's supplying three rough digitized points on each track with an image plane digitizer [...]. Two fiducial marks for scan table to FSD coordinate system reference are also digitized." ${ }^{17}$ Here we can see again that the digitization process is a combination of manual operations and specifically designed machines - that has to be traced in more detail.

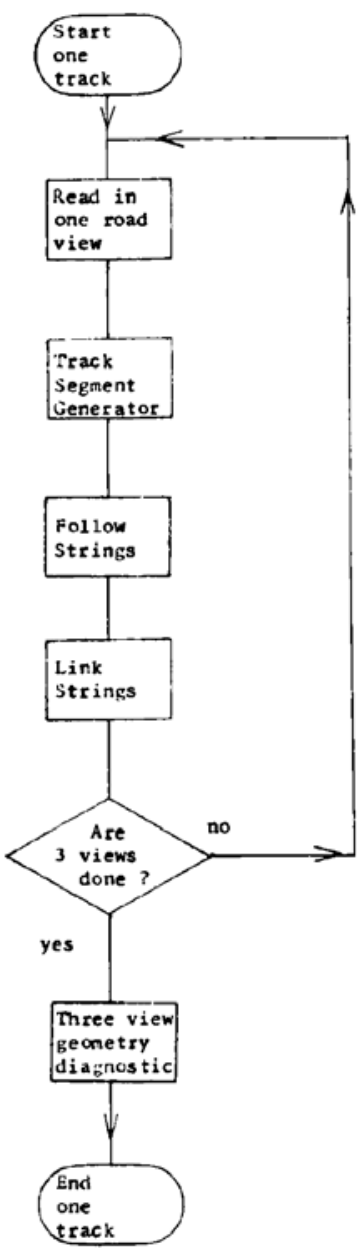

Fig. 6 Block Diagram

Block diagram for the pattern recognition of one bubble chamber track by the FILTER program, R. C. Strand, "Man-Machine Cooperation in Digital Pattern Recognition of Bubble Chamber Tracks," Annals of the New York Academy of Sciences 157, no. 1 (1967): 65-82 (77).

\footnotetext{
${ }^{17}$ Strand, Man-Machine Cooperation, 74/75.
} 
Fig. 6 shows a block diagram of software operations for the pattern recognition process. This software has to be analyzed more closely to understand more precisely how the construction of the pattern worked. I don't go in to more detail of this complicated processes here, since my paper is only meant to be a first sketch of a more detailed research project. Anyway: The pattern recognition done at Brookhaven Lab 1964 had to be stabilized over time: The mentioned image (Fig. 1), at the time of its production in 1964, was not yet recognized as the evidence we see it as today. More photographs had to be read as traces of the ' $\Omega$ ', , only this series stabilized the pattern recognition. ${ }^{18}$ Moreover results from other experiments had to be added. ${ }^{19}$ As further indications began to accumulate, the image was recognized as the first photo of an event during which an omega minus boson is produced and disintegrates. The photograph convinced most physicists that Murray Gell-Mann's theory was correct (he received the Nobel Prize in 1969) - today, it has become one of the supporting pillars in the so-called 'standard model of particle physics' under the heading 'quark model'.

Already in the 1960s in the then emerging 'standard model', the Higgs mechanism and its associated boson played a crucial role. ${ }^{20}$ They were postulated in a short paper, published coincidentally also in $1964 .{ }^{21}$ The boson was hypothetical for a long time and was demanded to explain the process of electroweak symmetry breaking. The detection of this particle - or at least of a very similar boson - was announced at CERN in 2012. Basically, the process was similar to the discovery of the Omega Minus: There was a theoretical model that predicted a particular particle. And now the task was to test whether or not this model correctly described nature (although it took a while until the need for a Higgs boson became clearer). How could the particle be found?

The search for the Higgs boson is the main example of a paper from Nature on the role of machine learning in particle physics. Interestingly, the text begins with a review of the

\footnotetext{
${ }^{18}$ See William B. Fowler and Nicholas P. Samios, “The Omega-Minus Experiment," Scientific American, Vol. 211, No. 4 (1964): 36-45 (42-45).

${ }^{19}$ It was for example important that experiments in "deep inelastic scattering", beginning around 1967, had shown protons as having a deeper structure which gave credibility to the idea of quarks being real physical entities and not only helpful mathematical constructions, see Jerome Friedman, "Deep-Inelastic Scattering and the Discovery of Quarks", in: Hoddeson et al., 566-588.

${ }^{20}$ See Brown et al., "The Rise of the Standard Model", 12/13 and 15/16.

${ }^{21}$ See Peter W. Higgs, "Broken Symmetries and the Masses of Gauge Bosons," Physical Review Letters Vol. 13, No. 16 (19. Oktober 1964), 508-509.
} 
bubble chambers and visual pattern recognition methods that we just discussed in relation to the ' $\Omega^{-}$'. I quote in length:

„Many known particles were discovered using detectors that made subatomic particles visible to the human eye. For example, bubble chambers filled with superheated liquids that boil when charged particles pass through them transform the paths of the particles into visible tracks of bubbles, which can then be photographed and analysed. The detectors at the Large Hadron Collider (LHC) are much more complex and record data at far greater rates than is possible using bubble chambers. For example, the $\mathrm{LHCb}$ experiment analyses as many events every six seconds as the Big European Bubble Chamber recorded in its entire 11 years of operation (1973-1983), and the datasets collected by the ATLAS and CMS experiments at the LHC are comparable to the largest industrial data samples. It is impossible for humans to visually inspect such large amounts of data; algorithms running on large computing farms took over this task long ago.“22

Obviously, the processing capabilities of human actors are no longer up to the gigantic amounts of data. There are now new detector technologies in operation, not any longer film recording bubble chamber events that then have to be painstakingly analyzed by manualstereoscopic-digital assemblages. The process is still very complex, but in different ways, which won't be described in detail here. ${ }^{23}$ Anyway: The mass and complexity of data is much higher:

"The sensor arrays of the LHC experiments produce data at a rate of about one petabyte per second. Even after drastic data reduction by the custom-built electronics used to readout the sensor arrays, which involves zero suppression of the sparse data streams and the use of various custom compression algorithms, the data rates are still too large to store the data indefinitely_ as much as 50 terabytes per second, resulting in as much data every hour as Facebook collects globally in a year.",24

\footnotetext{
${ }^{22}$ Alexander Radovic et al., "Machine Learning at the Energy and Intensity Frontiers of Particle Physics," Nature 560 (2018): 41-48 (41). For more technical details see Dimitri Bourilkov, "Machine and Deep Learning Applications in Particle Physics," https://arxiv.org/pdf/1912.08245.pdf.

${ }^{23}$ See Rudolf Frühwirth, Are Strandlie, Pattern Recognition, Tracking and Vertex Reconstruction in Particle Detectors (New York: Springer 2021). See esp. 169-180 on the CERN-detectors.

${ }^{24}$ Radovic et al., "Machine Learning", 41. The comparison to Facebook is interesting.
} 
This shows why particle physics is so interesting for any research in cultures of pattern recognition - because they deal with the most complex data. But why do these systems have to produce so much data?

"Einstein famously related mass $m$ to energy $E$ via $E=m c^{2}$, where $c$ is the speed of light in a vacuum. A powerful particle accelerator such as the LHC, which is $27 \mathrm{~km}$ in circumference, is therefore required to create particles orders of magnitude more massive than the proton, such as the Higgs boson. A Higgs boson is produced only once every few billion proton-proton collisions at the LHC. Many other interesting reactions occur orders of magnitude less often. To enable such data samples to be recorded in a reasonable time frame, the LHC collides nearly one billion protons per second." 25

It is necessary to create so many data on so many events because the processes you are looking for are so extremely rare - as the theory predicts.

"As stated above, a Higgs boson is produced only once every few billion proton-proton collisions at the LHC; however, the Higgs boson usually decays in ways that mimic much more copiously produced processes. The cleanest experimental signature of the Higgs boson involves its decay into two muon-antimuon pairs, which occurs roughly once every 10 trillion proton-proton collisions." ${ }^{26}$

That means the machine learning methods have above all the purpose to filter out the events which correspond to the predictions of the theory from the immense noise. It is very important to understand that the 'pattern recognition' is a completely different procedure in this case than it was in 1964. Fig. 7 shows how the data are 'focused' by machine learning to give a clear-cut peak: "BDTs are used to increase the resolution of the CMS electromagnetic calorimeter. When an electron or photon enters such a detector, it rapidly loses its energy, which is subsequently collected and measured by the calorimeter. This deposited energy is often recorded by many different sensors and the readings from these sensors must be clustered together to recover the original energy of the particle. Multivariate regression is used by CMS to train BDTs to provide corrections to these inferred energies on the basis

\footnotetext{
25 Ibid.

${ }^{26}$ Ibid.
} 
of all of the information contained in each calorimeter sensor." ${ }^{27}$ The pattern is no longer a structure, diagrammatically represented that stabilizes the meaning of a photograph full of traces. The pattern is a diagram that translates a very high amount of data into a visual representation - that has to be 'focused' ${ }^{28}$ In a way this can be read as the change from the image to the logic tradition as described by Galison. The immense amount of data produced by modern detectors leads also to new problems - often statistical fluctuations appear that seem to show a new particle or a new effect, but in the longer run, with more data, the effects disappear again - or they don't, in which a case a discovery can be declared. Or to put it another way: Patterns can be pseudo-patterns and there is a lot of work to be done to decide what is a legitimate pattern and to stabilize it.

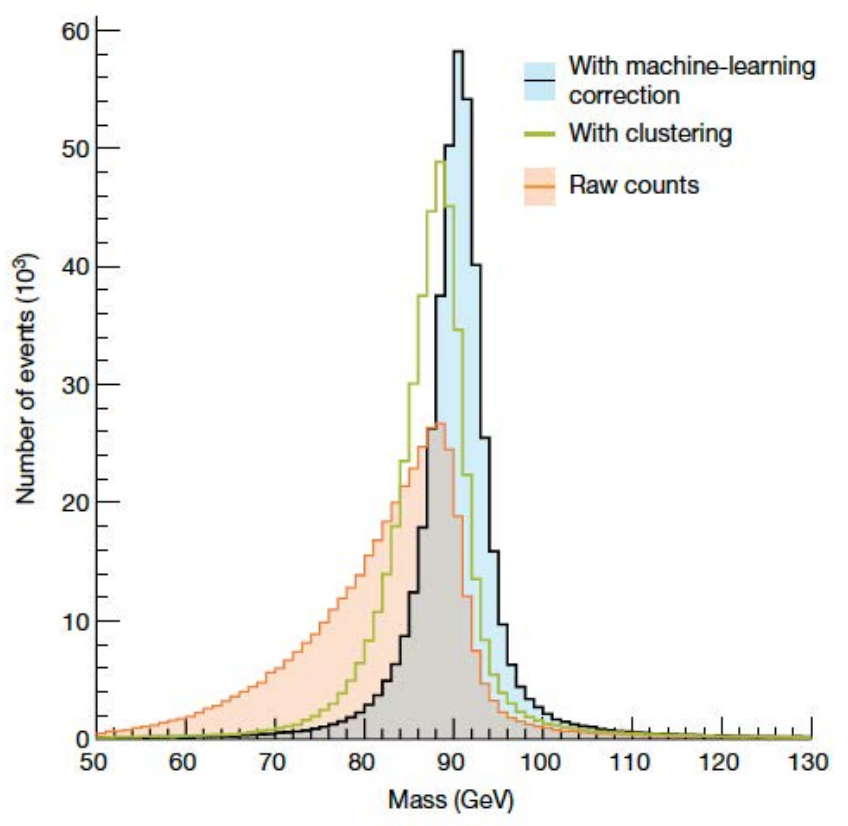

Fig. 1 | Machine learning for calorimetry at CMS. The mass distribution of $Z$ bosons that decay to electron-positron pairs $\left(Z \rightarrow e^{+} e^{-}\right)$, as measured in the central part of the CMS detector and binned into 1-GeV bins, is shown for three cases: using only the raw information from the detector (orange), after clustering the data (green) and after applying the machinelearning-based corrections discussed in the text (blue). The true position of the peak for this decay is $91 \mathrm{GeV}$. Image adapted from ref. ${ }^{101}$ under a CC BY 4.0 license, copyright CERN, reused with permission.

Fig. 7

Alexander Radovic et al., "Machine Learning at the Energy and Intensity Frontiers of Particle Physics," Nature 560 (2018): 41-48 (42)

\footnotetext{
${ }^{27}$ Ibid., 42. With 'BDT' 'boosted decision trees' are meant, a certain form of machine learning, see Shai ShalevSchwartz and Shai Ben-David, Understanding Machine Learning. From Theory to Algorithms (Cambridge: Cambridge University Press 2014), chapter 18.

${ }^{28}$ In the big data culture of contemporary particle physics the reproducibility of the experiment that was still given in the ' $\Omega$ '- -case is transformed. This transforms the 'culture of pattern recognition' too and has to be analysed in more detail.
} 
But how do the machine learning systems know which patterns to 'recognize'?

"The need to understand what signals will look like in the detectors and what other processes can mimic the signals has led to the development of high-quality simulation tools. Furthermore, the standard model provides accurate predictions of the rates and kinematic distributions of many of the processes that can mimic interesting signatures (referred to as backgrounds) and that contribute to particle-physics data samples, providing important benchmarks for validating the simulation tools and understanding their uncertainties. Therefore, simulated data samples are often used to train the machine-learning algorithms because in such samples all information is known by construction." 29

Even if this is simplified and would have to be investigated in much more detail: The analysis of the large amounts of data by the ML systems succeeds by training them with simulations according to the theory of the standard model (this is a little bit similar to the training of the female 'scanners' at Brookhaven, training them to detect interesting traces in pictures). Thus, the AI systems 'know' what to look for, so to speak. This shows that without the performative models of simulation, as Licklider had already called them in $1967^{30}$, data analysis would not work. Simulation stands between the theoretical model, let's say the theory of the Higgs-mechanism, and the 'raw data' (although this notion can only be used with caution). It is a three-step process of 'event generation', 'detector simulation' and 'event reconstruction'. ${ }^{31}$ Simulations perform the theory so that template-patterns can be generated that can be used to train the machine learning systems. Fig. 8 shows one representation of the results. An excess of events centered around the energy of $125 \mathrm{GeV}$ for a presumed decay channel Higgs to two $Z^{0}$-bosons, that each decay to two leptons $\left(\mathrm{e}^{+} \mathrm{e}^{-}\right.$or $\mu^{+} \mu^{-}$or $\left.\tau^{+} \tau^{-}\right)$.

\footnotetext{
${ }^{29}$ Ibid., 45.

${ }^{30}$ Cf. J. C. R. Licklider, "Interactive Dynamic Modeling," in Prospects for Simulation and Simulators of Dynamic Systems, ed. George Shapiro et al. (New York: MacMillan 1967), 281-289.

${ }^{31}$ See Michaela Massimi, Wahid Bhimji, "Computer Simulations and Experiments: The Case of the Higgs Boson," Studies in History and Philosophy of Science Part B: Studies in History and Philosophy of Modern Physics, Vol. 51, 2015, 71-81. See on the central role of computer simulation for science amongst others: Gabriele Gramelsberger, Computerexperimente: Zum Wandel der Wissenschaft im Zeitalter des Computers (Bielefeld: Transcript 2010) and Johannes Lenhard, Calculated Surprises. A Philosophy of Computer Simulation (Oxford: Oxford University Press 2019) and Franck Varenne, From Models to Simulations (London and New York: Routledge 2019), esp. chapter 8. See on the historical development of Monte Carlo-simulations in particle physics Galison, Image and Logic, chapter 8.
} 


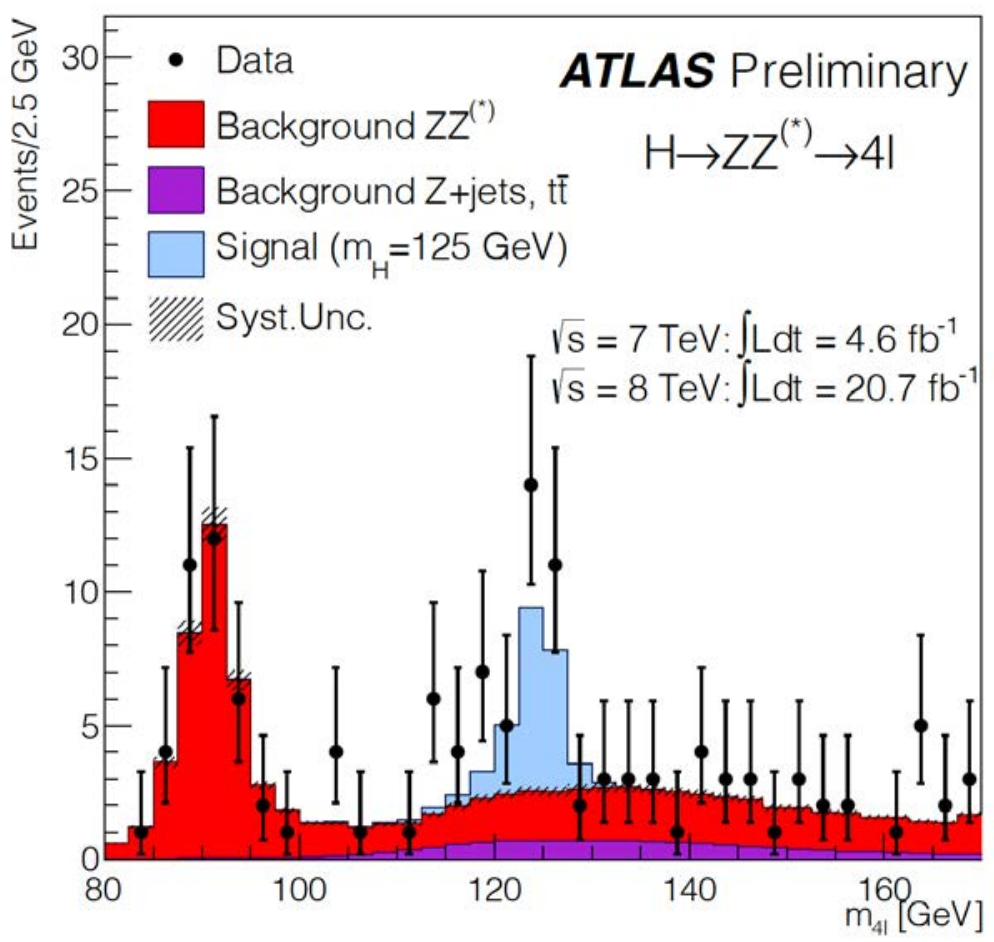

Fig. 8

From: The ATLAS Collaboration, Measurements of the properties of the Higgs-like boson in the four lepton decay channel with the ATLAS detector using $25 \mathrm{fb}^{-1}$ of proton-proton collision data, https://cds.cern.ch/record/1523699/files/ATLAS-CONF-2013-013.pdf, 15.

See Michaela Massimi, Wahid Bhimji, "Computer Simulations and Experiments: The Case of the Higgs boson," Studies in History and Philosophy of Science Part B: Studies in History and Phi-losophy of Modern Physics, Vol. 51, 2015, 71-81: "The black points are a count of the total events seen in each invariant-mass range in the actual physical experiment. The red and purple histograms are the expected number of background events determined from simulation as outlined in the description here below. The light blue histogram shows the expected distribution for a Higgs boson of a mass of $125 \mathrm{GeV}$ determined from simulated data."

AI in the sciences essentially means pattern recognition, especially through artificial neural networks and other forms of machine learning in huge amounts of highly complex data. There is continuity to past scientific practices that were also already pattern recognition in (photographic) data but with the difference that today we have a lot more data - the systems find patterns that humans can no longer recognize. The machine learning systems are in turn trained with data, but data that - at least in the example here - is itself generated by simulations, simulations that are in turn based on theoretical models. This should be 
emphasized because in 2008 Chris Anderson wrote a subsequently much-discussed, popular text in which it was claimed that the huge amount of data collected and its analysis by machine learning makes models and theory superfluous. Again a longer quote:

““All models are wrong, but some are useful.' So proclaimed statistician George Box 30 years ago, and he was right. But what choice did we have? Only models, from cosmological equations to theories of human behavior, seemed to be able to consistently, if imperfectly, explain the world around us. Until now. Today companies like Google, which have grown up in an era of massively abundant data, don't have to settle for wrong models. Indeed, they don't have to settle for models at all. [...] The big target here isn't advertising, though. It's science. The scientific method is built around testable hypotheses. These models, for the most part, are systems visualized in the minds of scientists. The models are then tested, and experiments confirm or falsify theoretical models of how the world works. This is the way science has worked for hundreds of years. Scientists are trained to recognize that correlation is not causation, that no conclusions should be drawn simply on the basis of correlation between $\mathrm{X}$ and $\mathrm{Y}$ (it could just be a coincidence). Instead, you must understand the underlying mechanisms that connect the two. Once you have a model, you can connect the data sets with confidence. Data without a model is just noise. But faced with massive data, this approach to science - hypothesize, model, test — is becoming obsolete. [...]."32

What Anderson claims here is, at least until today and at least in some sectors of science, wrong: Data analysis needs models and as one can see exactly at the LHC, it is still true: "Data without a model is just noise". The main task of the machine learning methods is to find the tracks predicted by the theory in the noise of the collision events - if they are found some people get Nobel prizes (as Higgs and Englert did in 2013). If the predicted events are not found or if completely different events are found, the theories are discarded or at least reformulated. 'Hypothesize, model, test' still applies. ${ }^{33}$ But perhaps, even in the age of Big Data, this persistence of theory and model applies only in this example. Maybe it is

\footnotetext{
32 Chris Anderson, "The End of Theory: The Data Deluge Makes the Scientific Method Obsolete," Wired, https://www.wired.com/2008/06/pb-theory/.

${ }^{33}$ See Valerie Hanson, "Intensification, Not Transformation: Digital Media's Effects on Scientific Practice," Science Transformed? Debating Claims of an Epochal Break, ed. Alfred Nordmann, Hans Radder and Gregor Schiemann (Pittsburgh: University of Pittburgh Press), 135-146. Her correct argument concerning digital media can be applied to the use of machine learning as well: More data, mores statistics instead of single images - but 'pattern recognition' still has to be done (in another way).
} 
different in other scientific fields and disciplines. Especially the use of Machine Learning in sociology, economics, indeed the humanities and cultural studies might operate quite differently - because do the latter make hypothetical predictions and design models that can be tested experimentally? Perhaps completely different patterns have to be recognized. Perhaps 'recognizing' means something different. Perhaps 'patterns' don't play the same role. These questions have to addressed in research on 'cultures of pattern recognition' - there is of course a lot of research on 'visual cultures' of science ${ }^{34}$ and even on 'data cultures ${ }^{35}$, but none on 'cultures of pattern recognition'. This remains to be done.

\footnotetext{
${ }^{34}$ See Klaus Hentschel, Visual Cultures in Science and Technology. A Comparative History (Oxford: Oxford University Press 2014), esp. chapter 9, which is called "Mastery of Pattern Recognition" and which is an important first step in the direction the research conceived in my paper.

${ }^{35}$ See the special issue of the Journal of Cultural Analytics, 2019 on "data cultures", several interesting articles, https://culturalanalytics.org/article/11053,
} 